\title{
Anemia como problema de saúde pública: uma realidade atual
}

\author{
Anemia as a public health problem: the current situation
}

M alaquias Batista Filho ${ }^{1}$

Ariani Impieri de Souza ${ }^{1}$

Cristiane Campello Bresani ${ }^{1}$

\footnotetext{
${ }^{1}$ Instituto M aterno Infantil Professor Fernando Figueira. Rua dos Coelhos 300, Boa Vista. 50070-550 Recife PE.

mbatista@imip.org.br
}

Abstract In 1990, the United Nations held a World Summit in New York, in which goals for the upcoming decadewereestablished. O ne of these goals was a one-third reduction in the prevalence of anemia among women at childbearing age. Despite this modest percentage, epidemiological indicators point to the opposite direction, indicating an increasing occurrence of anemia in different regions of the world, including in Brazil. These data show that anemia has continued since the antiquity to be one of themost prevalent and widespread diseases in human populations. Thus, a number of questions without answers arise re garding the actual prevalence and etiopathogenesis of the problem as well as with respect to the degree of implantation and the effectiveness of control measures.

Key words Anemia/diagnosis, Anemia/epidemiology
Resumo Em 1990, as N ações Unidas promove ram a Reunião de Cúpula de N ova Iorque, onde foram traçadas metas para o decênio vindouro, dentre as quais, a redução de um terço na prevalência das anemias nas mulheres em idade fértil. Porém, apesar de percentual modesto, indícios epidemiológi cos apontam no sentido inverso, ou seja, indicam a crescente e grave ocorrência de anemia em diferentes regiões do mundo, inclusive no Brasil. A o reunir esses informes, constata- se quea anemia continua, desde a antiguidade, a ser uma das entidades nosológicas mais prevalentes e difundidas nas populações humanas. A partir daí surge uma série de questionamentos ainda sem respostas em relação às reais prevalência e etiopatogenia do problema, ao grau de implantação e à efetividade das medidas de controle.

Palavras-chave Anemia/diagnóstico, Anemia/ epidemiologia 
Prevalência: crescente?

A anemia é uma das entidades nosológicas de descrição mais antiga na medicina e, provavelmente, uma das mais difundidas na humanidade. Dada a sua conotação pan-endêmica, tornaram-se incansáveis e repetitivas as citações da estimativa da Organização Mundial de Saúde (OMS), de que mais de dois bilhões de pessoas no mundo são anêmicas, correspondendo a um terço da população mundial ${ }^{1}$.

O Fundo das Nações Unidas pela Infância (UNICEF) foi ainda mais longe e previu que a anemia ferropriva chegaria a afetar até três biIhões e meio de indivíduos no mundo em desenvolvimento, contrastando com 853 milhões para a deficiência deiodo e 300 milhões para a deficiência de vitamina $A^{2}$. Todavia, o impacto das anemias e da deficiência de ferro (com ou sem anemia) na saúde coletiva deve ser apreciado não somente pela magnitudenumérica em termos epidemiológicos, mas também por suas conseqüências clínicas na saúde dos indivíduos afetados.

Sem dúvida, o caráter trans-social e pangeográfico representa o traço mais distintivo do quadro epidemiológico das anemias carenciais, quando comparadas com outros déficits nutricionais, tais como deficiência de iodo, hipovitaminose $\mathrm{A}$ e desnutrição energético-protéica ${ }^{1,2}$. N as duas últimas décadas, a anemia carencial, em especial a ferropriva, passou a ser reconhecida como a carência nutricional de maior prevalência no mundo, comportando-se como uma endemia de caráter cosmopolita, que se distribui em todos os continentes, blocos geoeconômicos e grupos sociais, embora sua ocorrência ainda conserve uma relação de dependência com a renda, a escolaridade, as condições insalubres das moradias e outras condições socioambientais negativas ${ }^{1,2,3}$.

De acordo com a OMS, pode-se classificar a significância populacional da prevalência deanemia como normal ou aceitável (abaixo de $5 \%$ ), leve (de 5 a 19,9\%), moderada (de 20 a 39,9\%) e grave (maior ou igual a 40\%). Em termos mundiais, a prevalência deanemia em paísesindustrializados éainda inaceitável, situando-se em torno de 5 a 16\%. Estimou-se que, entre os anos de 1990 e 1995, nos países desenvolvidos, a anemia acometeu $20 \%$ das crianças menores de cinco anos, $22 \%$ das gestantes, $10 \%$ das mulheres não grávidas, $4 \%$ dos homens e $12 \%$ dos idosos. Em contraponto, nos países em desenvolvimento, essas proporções seriam de 39\%, 52\%, 42\%, 30\% e $45 \%$, respectivamente, atingindo, portanto, ra- zões de prevalência de até 7,5 e traduzindo sua gravidade nesses locais ${ }^{1,2}$.

Numa perspectiva continental, a prevalência de anemia em mulheres não grávidas na ÍndiaÁsia é de $74 \%$ e na África, de $40 \%$. Na América Latina e Caribe, sabe-se que aproximadamente $30 \%$ das mulheres e $25 \%$ das crianças abaixo de cinco anos são anêmicas. No Brasil, segundo a OM S, 30\% das crianças abaixo de cinco anos têm anemia, sendo a segunda maior prevalência da América Latina ${ }^{4}$. Num enfoque nacional, al guns autores consideram que estas estimativas estariam subestimadas e que a prevalência efetiva do problema em crianças poderiaser $50 \%$ acima dos valores ditados pela OM S, alcançando $45 \%^{5,6}$.

Não existem no Brasil estudos para estimar de forma consistente a dimensão do problema, sobretudo em mulheres em idadefértil, cuja taxa seria em torno de $25 \%$, segundo uma revisão bibliográfica do período de 1990 a 20007. U m outro trabalho, sobre a transição nutricional no Brasil, realizado com base em três estudos transversais das décadas de 1970, 1980 e 1990, referencia o rápido declínio da prevalência de desnutrição em crianças e o crescimento, num ritmo mais acelerado, da prevalência de sobrepeso/obesidade em adultos, enquanto que as anemias continuam com prevalências elevadas, entre 40 e $50 \%$ em menores de cinco anos e 30 e $40 \%$ em gestantes. Este conclui que a anemia representa, em termos demagnitude, o principal problema carencial do país, aparentemente sem grandes diferenciações geográficas ${ }^{7-10}$.

Em Pernambuco, um inquérito estadual em 1997 estimou, de acordo com o critério da OMS (hemoglobina abaixo de $12 \mathrm{~g} / \mathrm{dL}$ ), prevalências deanemia de $46,7 \%$ entre as crianças menores de cinco anos e de $25 \%$ nas mulheres entre 10 e 49 anos (idade fértil). $\mathrm{Na}$ região metropolitana do Recife, o percentual deanemia para essas mulheres foi de $25,5 \%$; no interior urbano, de $19,9 \%$, e no interior rural, de $22,9 \%$, sendo as médias de hemoglobina (eos correspondentes DP) em cada uma dessas regiões, respectivamente de $13 \mathrm{~g} / \mathrm{dL}$ (1,69), 13,2 g/dL (1,56), 12,7 g/dL $(1,5)^{7,8,11}$.

\section{Etiopatogenia: estabel ecida?}

A deficiência de ferro, principalmente a alimentar, tem sido apontada como a causa mais comum de anemia, em proporções ainda não conhecidas de forma segura, mas que têm relação direta com a própria prevalência de anemia e 
variam entre as populações a depender da idade, do sexo, das condições socioeconômicas e da prevalência regional de outras causas de anemia, tais como malária, hemoglobinopatias hereditárias e deficiência de outros nutrientes (vitaminas A, B12, C eácido fólico) ${ }^{1}$.

A proporção atribuída à ferropeniaédas mais altas, sendo normalmente considerada a responsável pela grande maioria dos casos de anemia em populações de pobres condições socioeconômicas, entre as quais é baixa a ingestão de ferro biodisponível (produtos de origem animal) e/ou é comum a perda sangüínea devido à ancilostomíase ou esquistossomose, a exemplo do estado de Pernambuco, na região do Nordeste brasilei$\mathrm{ro}^{8}$. Nessas condições, mesmo quando outras causas deanemia são reconhecidas, a carência de ferro deveria estar presente como fator principal ou coadjuvante 1 . Dessa forma, a prevalência geral de anemia tem sido comumente utilizada como proxi da anemia ferropriva nas populações, mais apropriadamente naquelas que apresentam baixa freqüência das outras causas de queda na concentração de hemoglobina ${ }^{3}$. Em contrapartida, resultados do Global Burden of Disease Project 2000 da OM S permitem concluir que a ferropenia seria responsável por modestos $50 \%$ das anemias em termos mundiais ${ }^{12}$.

$N$ ão se pode esquecer que a anemia ferropriva é apenas o extremo mais grave do espectro evolutivo da carência de ferro. Baseando-se em dados do primeiro mundo, a prevalência estimada pela OMS de deficiência de ferro corresponderia a duas vezes e meia a prevalência de anemia ferropriva numa dada população ${ }^{1}$.

Ainda que a deficiência de ferro seja a carência de micronutriente mais comum emais disseminada no mundo e a única com prevalência também importante em praticamente todas as nações industrializadas, não existe nenhum quadro descritivo bem consolidado de sua situação global 1,3,4. Baseando-se no conceito de proxi eno critério matemático acima, poder-se-ia utilizar a anemia como um indicador indireto e extrapolar para outras populações, que não tivessem outras etiologias marcantes de anemia, a relação 1:2,5. Assim, poder-se ia estimar que a grande mai oria dos pré-escolares edas mulheres em idade fértil nos países menos desenvolvidos e, pelo menos 30 a 40\% nos países desenvolvidos, seriam ferrodeficientes ${ }^{1}$.

No entanto, a real distribuição etiológica das anemias nas diferentes populações não é conhe cida e alguns dados da literatura não coincidem com as estimativas da OM S; por outro lado, questionam-se a acurácia dos testes diagnósticosutilizados e de seus pontos de corte. Exemplificando, no grande inquérito americano Second $\mathrm{N}$ ational $\mathrm{H}$ ealth and $\mathrm{N}$ utrition Examination Survey (NHANES II), através da combinação de múltiplos exames, a prevalência de anemia e de anemia ferropriva foi inesperadamente baixa, respectivamente, $4 \%$ e $1,6 \%$ entremulheres de 20 a 44 anos $^{13}$. Por sua vez, Stoltzfus ${ }^{14}$, ao confrontar dados de diferentes inquéritos em al guns países em desenvolvimento, observou que as prevalências deanemia (hemoglobina abaixo de 11,0 $\mathrm{g} / \mathrm{dL}$ ) eram muito elevadas e semelhantes entre si; porém, quando se baixava o ponto de corte para $9,0 \mathrm{~g} / \mathrm{dL}$ ou menos, surgiram diferenças importantes que coincidiram com os graus de implantação dos programas de combate à carência de ferro.

\section{Anemia: uma doença negligenciada}

Ao lado de sua importância como evento epidemiológico, as anemias e, tipicamente, a ferropênica, configuram um problema de marcante expressão na prática clínica e de impacto socioeconômico. No primeiro aspecto, pode-se citar que normalmente as anemias carenciais vêm associadas a outras carências nutricionais, sobretudo no grupo infantil, equea ferropenia, mesmo sem anemia, pode malograr a homeostase de vários sistemas orgânicos. N o último aspecto, a deficiência de ferro atua nos custos públicos e privados da saúde, nas conseqüências sociais do aumento da mortalidade materna, na redução de produtividadeenas conseqüências em longo prazo no desenvolvimento mental ${ }^{1}$.

Em todos os estágios da vida, a condição em pauta pode interferir negativamente no funcionamento cognitivo, na capacidade física, na produção de hormônios tireoidianos e regulação da temperatura corporal eno estado imune, aumentando os riscos de infecções. $\mathrm{N}$ as pessoas produtivas, pode reduzir o rendimento no trabalho em $30 \%{ }^{1,2}$.

$M$ ais especificamente, a anemia no ciclo gestatório, especial mentepor carência deferro, pode estar associada a aumento da mortalidade materna, mortalidade perinatal, prematuridade, baixo peso ao nascer e morbidade do infante. Quarenta por cento de todas as mortes maternas perinatais estão ligadas à anemia $a^{1,15-17}$. Paralelamente, desfecho favorável da gravidez ocorre 
30 a 40\% menos em anêmicas e seus conceptos têm menos da metade da reserva normal de ferro, podendo apresentar maior freqüência de ane mia no primeiro ano de vida.

Apesar dessas e mais outras implicações na saúdecoletiva, o interesse dos governos pelo problema das anemias como tema de políticas públicas érelativamenterecente. Assim, só em 1990, com a realização da Reunião de Cúpula de N ova Iorque promovida pelas $\mathrm{N}$ ações Unidas, as anemias passaram a figurar no elenco das prioridades mundiais de saúde e nutrição e, ainda assim, com um peso bem modesto em relação a outros problemas menos prevalentes ${ }^{2,18}$.

As metas do UNICEF foram estipuladas em $33 \%$ de redução das anemias nas mulheres em idade fértil, enquanto que objetivaram o controle do bócio endêmico e da hipovitaminose $\mathrm{A}$ e a redução em $50 \%$ da prevalência da desnutrição infantil moderada e grave no decênio que se seguiria ${ }^{2,18}$. Provavelmente a intenção não era desavisada, mas envolvia inseguranças em se tentar o controle ou a erradicação do problema, tais como dificuldades na implantação dos planos de ação e a incerteza da real resposta à fortificação e à suplementação em larga escala comunitária (efetividade).

A subvalorização das anemias como proble ma de saúde pública em nível de políticas de governo de certa forma se reproduz no plano dos estudos científicos em escala epidemiológica ou clínica. 0 primeiro aspecto pode ser ilustrado levando-se em conta que, apesar das facilidades técnicas e dos custos relativamente baixos requeridos para a avaliação quantitativa do problema, ainda não se dispõe, na maioria das nações, inclusive no Brasil, de estudos efetivamente representativos sobre sua ocorrência, muito menos sobre o status populacional de ferro e sobre avaliação de implantação e impacto dos programas de controle?

Muito provavelmente, tal desinteresse seria explicado pelo aparente consenso de que o problema estaria esgotado como tema de investigação. Admite-se, de uma forma um tanto arbitrária, que a grande maioria das anemias decorre simplesmente da carência primária de ferro. Esta avaliação simplificada teoricamente satisfaz a compreensão da situação etioepidemiológica das anemias e das diretrizes estratégicas universais recomendadas para seu controle, como o enriquecimento das massas alimentares com ferro e folato, na presunção de que estariam cobertas suas duas grandes vertentes etiológicas sob o ponto de vista de carência nutricional primária.

\section{Estratégias de controle: 0 insucesso}

Em termos de melhora do status de ferro e de níveis de hemoglobina, a despeito da implementação dos programas de fortificação dos alimentos em muitos países, dispõe-se de poucas evidências de sua melhora na população, tanto pela falta de produção de dados, como pela não consolidação dos programas no que concerne à regulamentação, implementação, insumos e fiscalização ${ }^{4}$. Bastanteilustrativo éo caso das mulheres no ciclo grávido-puerperal, que ainda hoje expressam os mai oresíndices de anemia, mesmo após as recomendações de suplementação universal nesse estrato populacional ${ }^{1,2}$.

Um dos exemplos do insucesso da meta proposta para a década final do último século está citado no relatório da comissão técnica da divisão CARK (Central Asian Republics and e Kazakhstan) do UNICEF de 1996, queelevou o problema na região ao nível de "crise na saúde pública", exigindo medidas imediatas, diante das altas prevalências de anemia ferropriva em crianças (60\%) e mães ( $80 \%)$. Esse grupo de trabalho foi fase preliminar de um plano de ação nutricional regional integrada, a Estratégia de Controle e Prevenção de Anemia da CARK?

Outro indício da endemia écitado oficialmente no Relatório Regional para Prevenção e Controle da Anemia por D eficiência de Ferro em Mulheres e Crianças do UNICEF"OMS - Divisão da Europa Central e Oriental, Comunidade dos Estados Independentes e Países Bálticos, de 1999. Neste documento, estima-se que o problema afeta 40 a $50 \%$ das mulheres grávidas e $50 \%$ das crianças abaixo dos cinco anos na região, traçando eaconselhando estratégias regionais integradas para 0 enfrentamento da situação atual ${ }^{2}$.

Adicionalmente, o Global Burden of Disease Project 2000 da OM S, através de metanálises e de estimativas da OMS, traçou o impacto da anemia por deficiência deferro na saúde em termos mundiais, concluindo que 841.000 mortes e 35.057 .000 anos de vida incapacidade-ajustados (disability-adjusted life years - DALYs) são atribuíveis a essa doença e que a relação entre anemia materna e mortalidade perinatal é responsável por $56 \%$ dessas cifras ${ }^{12}$.

Nesse ponto, é importante conhecer a relação custo-benefício de cada intervenção de campo para o controle da carência de ferro. Se devidamente implantados, os programas de suplementação atingem uma efetividade de $70 \%$ em curto prazo com um custo dos componentes de ferro de apenas $7 \%$ do total do programa. Os 
programas gerais de fortificação de ferro são considerados $93 \%$ efetivos em longo prazo com $27 \%$ do custo total dos produtos manufaturados advindos do fortificante de ferro ${ }^{1}$.

Imaginando-se que, quando taxas de anemia estão acima de 30 a $40 \%$ num grupo idade-sexo definido, a maioria dos não anêmicos seria suficientemente ferropênica para estar sob risco de conseqüências funcionais adversas, medidas amplas para melhorar a nutrição de ferro dessa subpopulação são justificadas, até mesmo quando se considera a presença de outros fatores contribuintes para a anemia. E, teoricamente, nessa situação, seria mais efetiva e eficaz a suplementação universal deferro, enão o manejo caso a caso. A proposta de abordagem programática com suplementação seletiva efortificação alimentar éadequada para prevalências deanemia entre $5 \%$ e $20 \%$. Essa lógica é reforçada quando se assume que os trêsníveis de disponibilidadederecursos diagnósticos (pobre, intermediário e adequado) correspondem aos graus de prevalência de anemia ( grave, moderada e leve) para uma dada região $0^{1,3}$.

Essas recomendações, entretanto, parecem não fazer parte da política de saúde pública em muitos locais ${ }^{14}$. $E$, efetivamente, a limitação da meta para redução das anemias em 33\% restrita ao grupo de mulheres em idade fértil já se delineia uma proposição aquém da necessária. Po- rém, dianteda boa efetividadeestimada pela OM S para os programas de suplementação e fortificação, é possível ainda conjecturar que as respostas reduzidas obtidas com intervenções populacionais e experimentais possam, pelo menos em parte, ser atribuídas a al guns pressupostos ainda não devidamente consolidados, entre os quais se incluiria a caracterização etiopatogênica, morfofuncional e diagnóstica das anemias; inter-relação entre eficácia/efetividade e efeitos adversos/ adesão terapêutica; e o estágio de implantação dos programas de controle das anemias.

São lacunas que demandam respostas na busca do controle do problema e que poderão ser preenchidas pelas seguintes alternativas: realizar inquéritos populacionais para se determinar a distribuição das etiologias nos diversos povos de acordo com idade, sexo epeculiaridades fisiológicas; realizar estudos clínico-laboratoriais a fim de determinar a real participação da carência de ferro e a eficácia da reposição de ferro.

\section{Conclusão}

A pesar do seu impacto epidemiológico eclínico, a anemia ainda conserva através do tempo incertezas no que se refere a causas, epidemiologia, diagnóstico e tratamento. 


\section{Colaboradores}

Batista Filho M trabalhou na concepção, redação e revisão final do artigo. Souza Al trabalhou na concepção, redação e revisão final do artigo. Bresani CC trabalhou na concepção, revisão da literatura e redação do artigo.

\section{Referências}

1. World Health Organization. Iron deficiency anemia: assessment, prevention and control - a guide for programme managers. Geneva: WHO; 2001.

2. UNICEF/WHO. Prevention and control of iron deficiency anaemia in women and children. Geneva: UNICEF/WHO; 1999.

3. Souza Al, Batista Filho M. Diagnóstico e tratamento das anemias carenciais na gestação: consensos e controvérsias. Rev Bras Saúde M atern Infant 2003; 3(4):473-479.

4. Pan American Health Organization. Flour fortification with iron, folic acid and vitamin B12. Santiago: PAH O; 2003.

5. Monteiro CA, Szarfarc SC, M ondini L. Tendência secular da anemia na infância na cidade de São Paulo (1984-1996). Rev. Saúde Pública 2000; 34(Supl 6):62-72.

6. Batista Filho M, Migliolo TC. Alimentação e nutrição no nordeste do Brasil. Recife: Instituto $M$ aterno Infantil Professor Fernando Figueira; 2006.

7. Santos $L M P$, organizador. Bibliografia sobre deficiência de micronutrientes no Brasil: 1990-2000. Vol. 2. Brasília: OPAS; 2002.

8. Batista Filho M, Romani SAM. Alimentação, nutrição e saúde no estado de Pernambuco. Recife: Série de Publicações Científicas do Instituto M aterno Infantil de Pernambuco; 2002.

9. Batista Filho M, Rissin A. A transição nutricional no Brasil: tendências regionais e temporais. Cad Saúde Pública 2003; 19(Supl 1):181-191.

10. Ashworth A, Batista Filho M, Lira PIC, O sório M M . Prevalência de anemia em crianças de 6-59 meses de idade no estado de Pernambuco, Brasil. Rev Panam Salud Públ 2001; 10(2):101-107.

11. Silva $S C L$, Batista Filho M. Anemia em mães e filhos no estado de Pernambuco. Recife: Instituto M aterno Infantil Professor Fernando Figueira; 2005.

12. Stoltzfus RJ. Iron deficiency: global prevalence and consequences. Food N utr Bull 2003; 24(Supl 4):99103.

13. American Society for Clinical Nutrition. Summary of a report on assessment of iron nutritional status of United States population. Am J Clin Nutr 1985; 42:1318-1330.

14. Stoltzfus RJ. Rethinking anaemia surveillance. Lancet 1997; 349:1764-1766.

15. Rasmussen KM. Iron deficiency anemia: reexamining the nature and magnitude of the public health problem. Is there a causal relationship between iron deficiency or iron-deficiency anemia and weight at birth, length of gestation and perinatal mortality? J Nutr 2001; 131(Supl):590-603.

16. Allen LH. Anemia and iron deficiency: effects on pregnancy outcome. Am J Clin Nutr 2000; 71(Supl):1280-1284.

17. Scholl TO, Reilly T. Anemia, iron and pregnancy outcome. J Nutr 2000; 130(2): 443.

18. UNICEF. Estratégia para melhorar a nutrição de crianças e mulheres nos países em desenvolvimento. Nova York: UNICEF; 1990.

Artigo apresentado em 19/07/2007

Aprovado em 16/08/2007 